\title{
REGULAR GRAPHS AND STABILITY
}

\author{
D. A. HOLTON and DOUGLAS D. GRANT*
}

(Received 7 April 1973; revised 25 September 1974)

\begin{abstract}
We show that a graph $G$ is semi-stable at vertex $v$ if and only if the set of vertices of $G$ adjacent to $v$ is fixed by the automorphism group of $G_{v}$, the subgraph of $G$ obtained by deleting $v$ and its incident edges. This result leads to a neat proof that regular graphs are semi-stable at each vertex. We then investigate stable regular graphs, concentrating mainly on stable vertex-transitive graphs. We conjecture that if $G$ is a non-trivial vertex-transitive graph, then $G$ is stable if and only if $\Gamma(G)$ contains a transposition, offering some evidence for its truth.
\end{abstract}

\section{Introduction}

Throughout this paper, all graphs considered are finite, undirected and are without loops and multiple edges. If $G$ is such a graph, we denote by $V(G)$ its vertex set; unless otherwise specified, we shall assume $V(G)=\left\{v_{i}\right\}_{i=1}^{p}$. All basic graph-theoretical concepts used in this paper are defined in Behzad and Chartrand (1971), and we use the permutation group terminology of Wielandt (1964).

If $u$ and $v$ are adjacent vertices of $G$, we write $u \sim v$; otherwise we write $u \sim v$. If $u \in V(G)$, we define the open neighbourhood of $u, N_{G}(u)$, to be the set $\{v \in V(G): u \sim v\}$. The closed neighbourhood of $u, \overline{N_{G}(u)}$, is $N_{G}(u) \cup\{u\}$. We denote by $\operatorname{deg}_{G}(u)$, the degree of $u$ (that is, the cardinality of $\left.N_{G}(u)\right)$. For convenience, in these definitions we omit the subscript $G$ if no confusion can arise. If $W \subseteq V(G)$, then $G_{W}$ denotes the subgraph of $G$ induced by $V(G) \backslash W$. For convenience, if $W$ consists of a single vertex $v$, we write $G_{v}$ rather than $G_{\{v\}}$. Denoting the automorphism group of $G$ by $\Gamma(G)$, we define $\Gamma(G)_{W}$ to be the subgroup of $\Gamma(G)$ all of whose elements fix each element of $W \subseteq V(G)$; here, contrary to the usual convention, we consider $\Gamma(G)_{W}$ to act only on $V(G) \backslash W$. If $W=\{v\}$, we write $\Gamma(G)_{v}$ rather than $\Gamma(G)_{\{v\}}$.

We say that $G$ is semi-stable at $v \in V(G)$ if $\Gamma\left(G_{v}\right)=\Gamma(G)_{v}$, (see Holton (1973a)). Thus $G$ is semi-stable at $v$ if and only if every automorphism of $G_{v}$ ex-

* The research of the second author was supported by a Commonwealth Postgraduate Research Award. 
tends to an automorphism of $G$. We say that $G$ is stable if there exists a sequence $S$ of all the vertices of $G$ which, by relabelling of $V(G)$ if necessary, we can assume to be $\left\{v_{1}, v_{2}, \cdots, v_{p}\right\}$, such that $\Gamma\left(G_{S_{j}}\right)=\Gamma(G)_{s_{j}}$ for $j=1,2, \cdots, p$, where $S_{j}=\left\{v_{1}, v_{2}, \cdots, v_{j}\right\}$. We say that $S$ is a stabilising sequence for $G$. The concept of stability is not to be confused with the similarly named, related, but different concept of Sheehan (1972).

Here we show that $G$ is semi-stable at $v$ if and only if $N_{G}(v)$ is fixed by $\Gamma\left(G_{v}\right)$, and use this result to generalise the theorem of Holton (1973a) that all regular graphs are semi-stable at each of their vertices. We then show that if $G$ is a nontrivial stable graph, then $\Gamma(G)$ contains a transposition, this result having been used before, Holton (1973b), but not proved. This necessary condition for stability, which was shown in Holton (1973b) to be also sufficient if $G$ is a tree, is shown by means of examples not to be sufficient if $G$ is a regular graph. Consequently, we attempt to prove that it is sufficient if $G$ is a vertex-transitive graph. This necessitates the characterisation of vertex-transitive graphs whose automorphism groups contain a transposition, which we accomplish. Unfortunately, we cannot resolve whether or not the possession by $\Gamma(G)$ of a transposition is sufficient for the vertex-transitive graph $G$ to be stable, but we obtain several subsidiary results which suggest that this might be true, and indicate the directions in which research on the problem might be pursued.

\section{A characterisation of semi-stability}

In this section we present a characterisation of semi-stability. We use the following lemma of Holton (1973a).

LEMMA 1. If $v \in V(G)$, then $\Gamma(G)_{v} \leqq \Gamma\left(G_{v}\right)$.

THEOREM 2. (The characterisation). $G$ is semi-stable at $v$ if and only if $N_{G}(v)$ is fixed by $\Gamma(G)$.

Proof. If $v$ is an isolated vertex of $G$, then trivially $\Gamma\left(G_{v}\right) \leqq \Gamma(G)_{v}$, and, as $N_{G}(v)=\varnothing, N_{G}(v)$ is fixed by $\Gamma\left(G_{v}\right)$. Thus suppose henceforth that $N_{G}(v) \neq \varnothing$.

(i) Assume $G$ is semi-stable at $v$. Let $g \in \Gamma(G)$, with $v^{g}=v$, and let $g^{\prime} \in \Gamma(G)_{v}$ be the restriction of $g$ to $V(G) \backslash\{v\}$. Now, if $u \sim v$ in $G$, it follows that $u^{g^{\prime}} \sim v$ in $G$. Hence $N_{G}(v)$ is fixed by $g^{\prime}$. We deduce that $N_{G}(v)$ is fixed by $\Gamma(G)_{v}$. However, as $G$ is semi-stable at $v, \Gamma(G)_{v}=\Gamma\left(G_{v}\right)$, so that $N_{G}(v)$ is fixed by $\Gamma\left(G_{v}\right)$.

(ii) Assume $N_{G}(v)$ is fixed by $\Gamma\left(G_{v}\right)$. Let $h \in \Gamma\left(G_{v}\right)$, and let $h^{*}=h(v)$ be the corresponding permutation on $V(G)$ which fixes $v$. We show that $h^{*} \in \Gamma(G)$. Let $u_{1}, u_{2}$ be adjacent vertices of $G_{v}$. As $h \in \Gamma\left(G_{v}\right)$, it follows that $u_{1}^{h^{*}} \sim u_{2}^{h}$. Thus $u_{1}^{h^{*}} \sim u_{2}^{h^{*}}$. Now let $u \in N_{G}(v)$, so that $u \sim v$ in $G$. By hypothesis, $N_{G}(v)$ is fixed by $\Gamma\left(G_{v}\right)$, so that $u^{h}=w$ for some $w \in N_{G}(v)$. It follows that $u^{\wedge}=w$. Now $v^{h^{*}}=v$, so that the edge $(u, v)$ of $G$ is mapped onto the edge $(w, v)$ of $G$ by $h^{*}$. We 
deduce that $h^{*}$ preserves adjacency in $G$. As $G$ is a finite graph, it follows immediately that $h^{*} \in \Gamma(G)$. Consequently, $h \in \Gamma(G)_{v}$, so that $\Gamma\left(G_{v}\right) \leqq \Gamma(G)_{v}$. By Lemma $1, \Gamma(G)_{v} \leqq \Gamma\left(G_{v}\right)$. We deduce that $\Gamma\left(G_{v}\right)=\Gamma(G)_{v}$, and that $G$ is semi-stable at $v$.

The importance of this characterisation is that to decide whether or not $G$ is semi-stable at $v$ we need only decide whether or not $N_{G}(v)$ is fixed by $\Gamma\left(G_{v}\right)$. This in many cases is an easier task than deciding whether or not $\Gamma\left(G_{v}\right)=\Gamma(G)_{v}$. As an example of its power, we prove a generalisation of Holton's result (1973a), that all regular graphs are semi-stable at each of their vertices, that is completely semi-stable. We require a definition. The graph $G$ is congruence-regular if there exists an integer $n>1$ and a non-negative integer $m<n$ such that $\operatorname{deg}_{G}(v) \equiv m$ $(\bmod n)$ for all $v \in V(G)$. We have:

THEOREM 3. All congruence-regular graphs are completely semi-stable.

ProOF. Let $G$ be a congruence-regular graph, such that for $n>1$ and $0 \leqq m<n, \operatorname{deg}_{G}(u) \equiv m(\bmod n)$ for all $u \in V(G)$. Let $v \in V(G)$. In $G_{v}$, if $w \in N_{G}(v)$, then $\operatorname{deg}_{G_{v}}(w) \equiv(m-1)(\bmod n)$, whereas if $w \notin N_{G}(v)$, then $\operatorname{deg}_{G_{v}}(w) \equiv m$ $(\bmod n)$. It follows that $N_{G}(v)$ is fixed by $\Gamma\left(G_{v}\right)$, so that, by Theorem $2, G$ is semi-stable at $v$. The theorem follows.

Immediate is the following corollary, which includes Holton's result.

Corollary. (a) All regular graphs are completely semi-stable.

(b) All Eulerian graphs are completely semi-stable.

Proof. (a) If $G$ is regular of degree $r$, we can choose $n=r, m=0$ and use Theorem 3.

(b) If $G$ is Eulerian, then by Theorem 3.1 of Behzad and Chartrand (1971), we can choose $n=2, m=0$ and apply Theorem 3 .

Theorem 3 suggests the following definition. A semi-stable extension of a graph $G$ is a graph obtained from $G$ by adding a new vertex $v$ and the edges $\left(v, v_{i}\right), i=1,2, \cdots, n$, where the subset $\left\{v_{i}\right\}_{i=1}^{n}$ of $V(G)$ is fixed by $\Gamma(G)$. Theorem 3 can thus be interpreted as: $G$ is semi-stable at $v$ if and only if $G$ is a semi-stable extension of $G_{n}$. We shall use the notion of a semi-stable extension in the next section.

\section{Some aspects of stable regular graphs}

In Holton (1973b), the following quite simple result was used, without proof. We now supply a short proof.

THEOREM 4. If $G$ is stable, then either $G$ is $K_{1}$ or $\Gamma(G)$ contains a transposition.

Proof. $K_{1}$ is stable. Assume $|V(G)|=p>1$ and let $S=\left\{v_{1}, v_{2}, \cdots, v_{p}\right\}$ be 
a stabilising sequence for $G$. Now $G_{S_{p-2}}$ is either $K_{2}$ or $K_{2}$, so that $\left(v_{p-1} v_{p}\right) \in$ $\Gamma\left(G_{S_{p-2}}\right)$. As $G$ is stable, $\Gamma(G)_{S_{p-2}}$ contains $\left(v_{p-1} v_{p}\right)$. It follows that $\Gamma(G)$ contains the transposition $\left(v_{1}\right)\left(v_{2}\right) \cdots\left(v_{p-2}\right)\left(v_{p-1} v_{p}\right)$.

Holton (1973b) showed that if $G$ is a tree and $\Gamma(G)$ contains a transposition, then $G$ is stable. McAvaney, Grant and Holton (1974) showed that a similar result holds if $G$ is unicyclic. It is not true, however, that if $G$ is regular and $\Gamma(G)$ contains a transposition then $G$ is stable. For example, the graph $G$ of Figure 1 is regular of degree 2, and its automorphism group contains the transposition $\left(v_{1} v_{2}\right)$. However, $G$ is $K_{3} \cup C_{5}$, and so by Theorem 5 of Holton (1973) is stable if and only if both $K_{3}$ and $C_{5}$ are stable. However, $\Gamma\left(C_{5}\right)$ does not contain a transposition, so that $C_{5}$ is not stable. It follows that $G$ is not stable. We can clearly construct several other examples of this type. By the above argument,
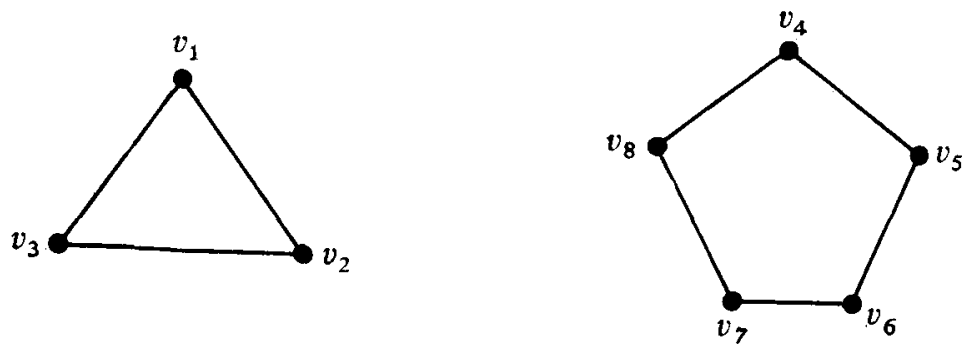

Fig. 1

if $G$ is a stable regular graph, all components of $G$ are stable regular graphs. Thus to find all stable regular graphs, it is sufficient to find all connected stable regular graphs. Moreover, as either a graph or its complement is connected, we need only find all stable regular graphs $G$ which are complement-connected (that is both $G$ and $\bar{G}$ are connected).

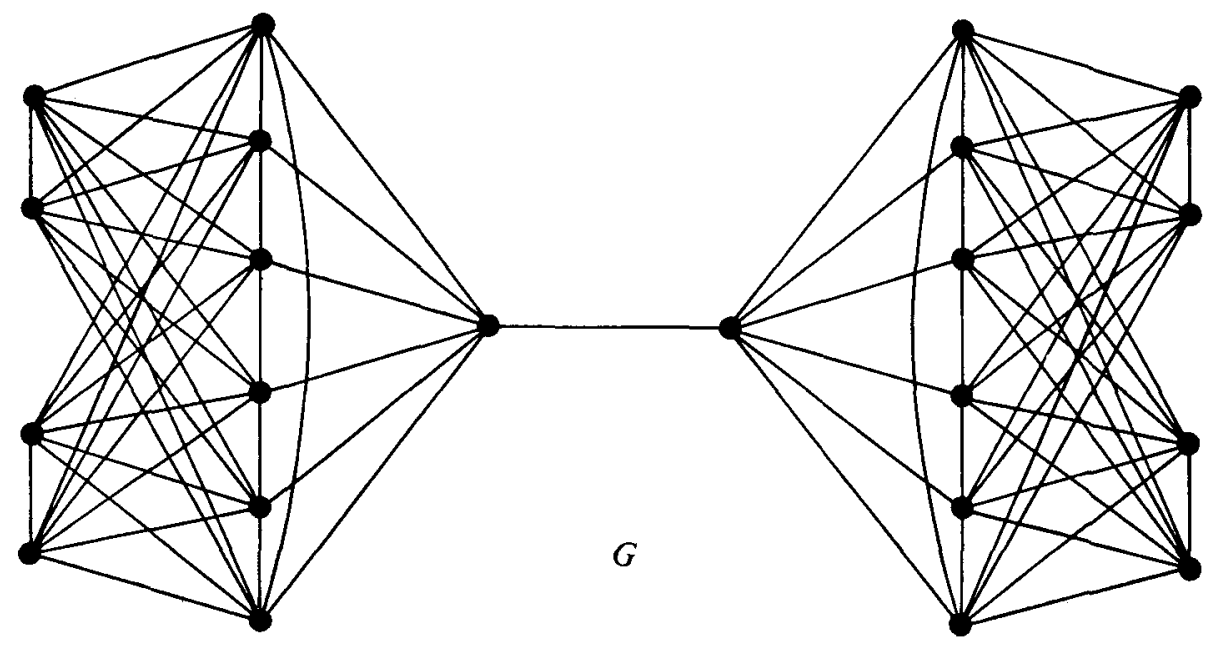

Fig. 2 
In Figure 2 we provide an example of a complement-connected regular graph $G$, such that $\Gamma(G)$ contains a transposition, which is not stable. We can readily construct an infinite number of such graphs. It thus remains a difficult problem to characterise complement-connected stable regular graphs. Below we make a start on the investigation of this problem.

We say that $G$ is vertex-transitive if $\Gamma(G)$ is a transitive group. Clearly if $G$ is vertex-transitive, then $G$ is regular. We now proceed to investigate the

CONJECTURE. If $G$ is vertex-transitive and $\Gamma(G)$ contains a transposition, then $G$ is stable.

Our first task is to characterise vertex-transitive graphs whose automorphism group contains a transposition. We need three preliminary lemmas, which we state without proof.

Lemma 5. Let $u, v \in V(G)$. Then $(u v) \in \Gamma(G)$ if and only if (i) in the case that $u \sim v, \overline{N_{G}(u)}=\overline{N_{G}(v)}$, (ii) in the case that $u \sim v, N_{G}(u)=N_{G}(v)$.

Lemma 6 (Wielandt (1964), Theorem 13.3). If $P$ is a primitive permutation group containing a transposition, then $P$ is a symmetric group.

Lemma 7. If $|V(G)|=p$, then $\Gamma(G)=\mathscr{S}_{p}$ if and only if $G$ is $K_{p}$ or $\bar{K}_{p}$. (Here $\mathscr{S}_{p}$ is the symmetric group of degree $p$ ).

Define $\boldsymbol{F}$ to be the family of all graphs $G$ such that (i) $G$ is vertex-transitive and (ii), if $(u v) \in \Gamma(G)$, then $u \sim v$. Recall that if $G_{1}$ and $G_{2}$ are graphs, then $G_{1}\left[G_{2}\right]$ denotes the composition of $G_{1}$ round $G_{2}$ (see Harary (1969), page 22). We have:

THEOREM 8.: If $G$ is vertex-transitive and $\Gamma(G)$ contains a transposition, then $G$ is of the form $H\left[K_{n}\right]$ or $H\left[\bar{K}_{n}\right]$ for some $n>1$, where $H \in \boldsymbol{F}$. Conversely, if $G$ is such a graph, then $G$ is a vertex-transitive graph whose automorphism group contains a transposition.

Proof. (i) Let $G$ be vertex-transitive, and suppose $u, v \in V(G)$ are such that $(u v) \in \Gamma(G)$. By Lemma 6, if $\Gamma(G)$ is primitive, then $\Gamma(G)$ is a symmetric group, and so by Lemma 7, either $G$ or $\bar{G}$ is a complete graph, and the theorem holds with $H=K_{1}$. Now suppose that $\Gamma(G)$ is imprimitive, and let $H_{1} \subset V(G)$ be a minimal non-trivial block of $\Gamma(G)$, of cardinality $n>1$. Let $H_{1}, H_{2}, \cdots, H_{m}$ be the complete block system of $\Gamma(G)$ containing $H_{1}$. Denote by $\left\langle H_{i}\right\rangle$ the subgraph of $G$ induced by $H_{i}$. As $G$ is vertex-transitive, the $\left\langle H_{i}\right\rangle$ are all isomorphic. Moreover, if $\Gamma_{i}$ denotes the restriction of $\Gamma(G)$ to $H_{i}$, then $\Gamma_{i}$ is transitive, is a subgroup of $\Gamma\left(\left\langle H_{i}\right\rangle\right)$, and, by the minimality of $H_{1}$, is primitive. Now suppose $u \in H_{j}$ and $v \in H_{k}, k \neq j$. As $(u v) \in \Gamma(G)$, it follows from the fact that $H_{j}$ and $H_{k}$ are blocks that $\left|H_{j}\right|=\left|H_{k}\right|=n=1$, a contradiction. Thus if $u \in H_{j}$, 
then $v \in H_{j}$. We deduce that $(u v) \in \Gamma_{j}$. By Lemma $6, \Gamma_{j}$ is symmetric, so that $\Gamma_{j}=\Gamma\left(\left\langle H_{j}\right\rangle\right)$ and by Lemma $7,\left\langle H_{j}\right\rangle$ is either $K_{n}$ or $R_{n}$. It follows that either $\left\langle H_{i}\right\rangle=K_{n}$ for all $i$ or $\left\langle H_{i}\right\rangle=\bar{K}_{n}$ for all $i$.

Let $a$ and $c$ be adjacent vertices of $G$ such that $a \in H_{j}$ and $c \in H_{k}, k \neq j$. Let $d \in H_{j} \backslash\{a\}$. As $G$ is vertex-transitive and $\Gamma(G)$ contains a transposition, we may suppose using the above argument, that there is $b \in H_{j} \backslash\{a\}$ such that $(a b) \in \Gamma(G)$. If $b \neq d$, then $(b d) \in \Gamma_{j}$. Let $g$ be a permutation in $\Gamma(G)$ whose restriction to $\Gamma_{j}$ is $(b d)$. It follows that $g^{-1}(a b) g=(a d) \in \Gamma(G)$. Thus in all cases $(a d) \in \Gamma(G)$. We deduce by Lemma 5 , that $d \sim c$. It follows that each vertex in $H_{j}$ is adjacent to each vertex in $H_{k}$, so that $G$ is in fact $H\left[K_{n}\right]$ or $H\left[R_{n}\right]$ for some graph $H$. If $H$ were not vertex-transitive, then for some $p$ and $q$ there would be no automorphism of $G$ mapping $H_{p}$ onto $H_{q}$, whence $G$ would not be vertextransitive. Moreover, if $H$ were not in $\boldsymbol{F}$, then for some $r$ and $s$, for all $w \in H_{r} \cup H_{s}, \overline{N_{G}(w)}$ would be the same, so by Lemma 5, there would exist $x \in H_{r}$ and $y \in H_{s}$ such that $(x y) \in \Gamma(G)$, contradicting our previous conclusion. It follows that $H \in \boldsymbol{F}$.

(ii) The converse result follows by noting that if $u$ and $v$ are vertices in the same copy of $K_{n}$ in $G=H\left[K_{n}\right]$, then $\bar{N}_{G} \overline{(u)}=\overline{N_{G}(v)}$, so that by Lemma 5 , $(u v) \in \Gamma(G)$, and that a similar result holds for $H\left[K_{n}\right]$.

Thus to prove our conjecture, we need only show that graphs of the type $H\left[K_{n}\right]$ and $H\left[\widehat{K}_{n}\right]$ for $H \in \boldsymbol{F}$ are stable. We now indicate that in fact we need not examine all such graphs.

First of all, from the definition of the composition of two graphs we can deduce the following two lemmas.

Lemma 9. $\overline{G_{1}\left[G_{2}\right]}=\bar{G}_{1}\left[\bar{G}_{2}\right]$.

LEMMA 10. If $n=p q$, where $p$ and $q$ are integers greater than 1 , then $H\left[K_{n}\right]=\left(H\left[K_{p}\right]\right)\left[K_{q}\right]$.

In Grant (to appear), the following result was proved.

LEMMA 11. If $G$ is stable, then $G\left[K_{n}\right]$ is stable for all $n>1$.

Finally, Holton (1973) proved

Lemma 12. $G$ is stable if and only if $\bar{G}$ is stable.

We deduce

THEOREM 13. If the conjecture is true for all graphs of the form $H\left[K_{p}\right]$ where $p$ is prime and $H$ is a vertex-transitive graph whose automorphism group contains no transpositions, then it is true a conjecture.

Proof. Assume the conjecture holds for all graphs of the form stated. We shall show by induction that it holds for all graphs given by Theorem 8 . It is 
readily observed that such graphs with few vertices are stable. (We omit the details). Suppose that $G=H\left[K_{n}\right]$ or $H\left[K_{n}\right]$, where $H \in F$ and $\Gamma(H)$ contains a transposition. In the former case, we may suppose by the inductive hypothesis that $H$ is stable, so that $G$ is stable by Lemma 11 . In the latter, $\bar{G}$ is $\bar{H}\left[K_{n}\right]$. As $\Gamma(H)=\Gamma(\bar{H})$, it follows that $\bar{H}$ is vertex-transitive and that $\Gamma(\bar{H})$ contains a transposition. As above, $\bar{H}$ is stable, so that $\bar{G}$ is stable. By Lemma $12, G$ is stable. Now suppose that $G=H\left[K_{n}\right]$ or $H\left[\bar{K}_{n}\right]$ where $H$ is vertex-transitive, but $\Gamma(H)$ does not contain a transposition. In the latter case, $\bar{G}=\tilde{H}\left[K_{n}\right]$, where $\bar{H}$ is vertex-transitive, but $\Gamma(\bar{H})$ does not contain a transposition. By Lemma 12, it follows therefore, that we need once again only consider the former case, as the latter is contained in it. We may suppose that $n$ is composite, and that $p$ is the smallest prime dividing $n$. Let $q=n / p$. By Lemma $10, G=\left(H\left[K_{p}\right]\right)\left[K_{q}\right]$. $\Gamma\left(H\left[K_{p}\right]\right)$ contains a transposition, and $H\left[K_{p}\right]$ is certainly vertex-transitive, so that by the inductive hypothesis, $H\left[K_{p}\right]$ is stable. It follows from Lemma 11 that $G$ is stable. This completes the proof.

To conclude, we offer the following result which supports our conjecture. We construct its proof using semi-stable extensions, but note that it is just as easy to obtain a proof by directly constructing a stabilising sequence.

THeOREM 14. $C_{m}\left[K_{n}\right]$ is stable for all $m \geqq 3, n \geqq 2$.

Proof. $C_{3}\left[K_{2}\right]=K_{0}$, and $K_{6}$ is stable. We thus assume $m+n>5$. In Figure 3, below, the symbol in (a) denotes a copy of $K_{n}$ and a dot (see vertex $\left.v_{3,1}\right)$ a single vertex. A line joining two symbols and/or dots indicates that each vertex represented by one symbol and/or dot is adjacent to each vertex represented by the other. We denote the copies of $K_{n}$ by $D_{1}, D_{2}, \cdots, D_{m}$ and the vertices in $D_{i}$ by $v_{i, 1}, v_{i, 2}, \cdots, v_{i, n}$. As $K_{n}$ is stable, we can clearly start with a copy $D_{1}$ of $K_{n}$ (see (a)). By adding the isolated vertex $v_{3,1}$, as in (b), we produce, by definition, a semi-stable extension of $K_{n}$. Since the set of all vertices in this graph is fixed by its automorphism group, we can add vertex $v_{2,1}$, and join it to $v_{3,1}$ and to each vertex in $D_{1}$, so that the graph in (c) is a semi-stable extension of that in (b). By a number of semi-stable extensions, we next build up copy $D_{2}$ of $K_{n}$, successively adding vertices $v_{2,2}, v_{2,3}, \cdots, v_{2, n}$ (see (d) and (e)). After adding vertex $v_{4,1}$, joined to $v_{3,1}$ (see (f)), we proceed as in (d), (e) and (f) until we reach (g). We then build up copy $D_{m-1}$ of $K_{n}$ (see (h)). Finally, we add successively the vertices $v_{m, 1}, v_{m, 2}, \cdots, v_{m, n}$, joining $v_{m, i}$ to all vertices in $D_{1}$ and $D_{m-1}$, and to $v_{m, j}$ for $j<i$ (see (i) and (j)). We obtain the graph $C_{m}\left[K_{n}\right]$, which is therefore stable. The stabilising sequence we construct is $\left\{v_{m, n}, v_{m, n-1}, \cdots, v_{m, 1}, v_{m-1, n}, \cdots, v_{m-1,2}, v_{m-2, n}\right.$, $\cdots, v_{m-2,2}, v_{m-1,1}, v_{m-3, n}, \cdots, v_{m-3,2}, v_{m-21}, v_{m-4, n}, \cdots, v_{4,1}, v_{2, n}, \cdots, v_{2,1}, v_{3,1}$, $\left.v_{1, n}, \cdots, v_{1,1}\right\}$.

COROLlaRY. $P_{k}\left[K_{n}\right]$ is stable for all $k \geqq 2, n \geqq 2$. 
Proof. $P_{2}\left[K_{2}\right]=K_{4}$, and is stable. For $k+n>4$, we note that at step (h) in Figure 3 , putting $m-1=k$, we have the graph $P_{k}\left[K_{n}\right]$, which is therefore stable.

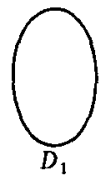

(a)

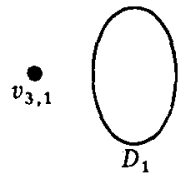

(b)

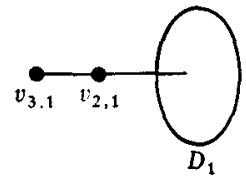

(c)

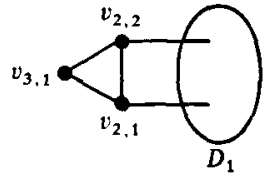

(d)

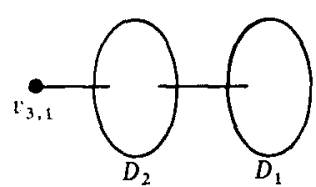

(e)

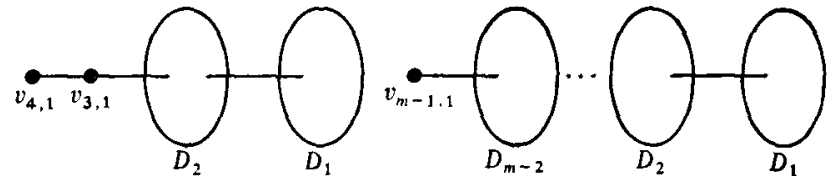

(g) (f)

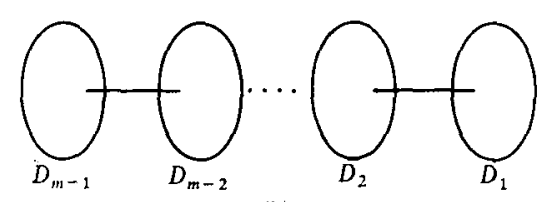

(h)

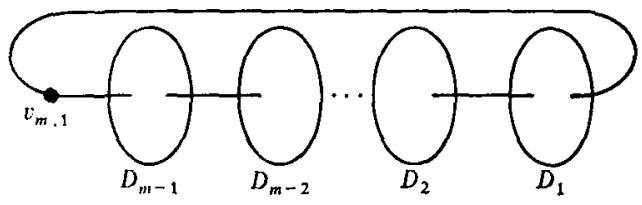

(i)

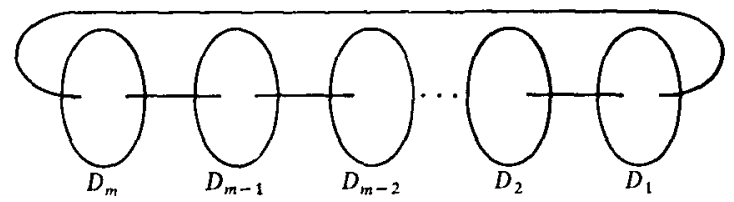

(j)

Fig. 3

\section{References}

M. Behzad and G. Chartrand (1971), Introduction to the Theory of Graphs (Allyn and Bacon (Boston), 1971).

F. Harary (1969), Graph Theory (Addison Wesley Reading, Mass. 1969).

D. A. Holton (1973), 'A Report on Stable Graphs', J. Austral. Math. Soc. 15, 163-171.

D. A. Holton (1973a), 'Two Applications of Semi-stable Graphs', Discrete Math. 4, 151-158.

D. A. Holton (1973b), 'Stable Trees', J. Austral. Math. Soc. 15, 476-481.

D. D. Grant (to appear), 'Stability and Operations on Graphs', Proc. Third Australian Conference on Combinatorial Mathematics.

K. L. McAvaney, D. D. Grant and D. A. Holton (1974), 'Stable and Semi-stable Unicyclic Graphs, Discrete Math. 9, 277-288.

J. Shechan, (1972), 'Fixing Subgraphs', J. Comb. Th 12A, 226-243.

H. Wielandt (1964), Finite Permutation Groups (Academic Press, New York, 1964).

University of Melbourne

Parkville Victoria 3052

Australia. 